\title{
Early Detection of Voltage Imbalances in Three-Phase Induction Motors $\dagger$
}

\author{
Rohan Samsi \\ samsi@psu.edu
}

\author{
Venkatesh Rajagopalan \\ vxr139@psu .edu
}

The Pennsylvania

University Park, PA 16802
Jeffrey Mayer

Asok Ray

axr2@psu .edu

mayer@ee.psu.edu

\begin{abstract}
Online Monitoring of induction motor health is of increased interest, as the industrial processes that depend on the motors become more complex and as performance to cost ratio of monitoring technology (e.g. sensors, microprocessors) has increased dramatically. Much efforts have been directed towards developing methods that use conventional Signal Processing and Pattern Classification techniques. This paper proposes a novel technique for early detection of stator voltage imbalances in three-phase induction motors, which is built upon the theories of wavelet transforms and symbolic time series analysis.
\end{abstract}

\section{INTRODUCTION}

The high levels of automation and process integration in modern manufacturing facilities place stringent demands on the reliability of large numbers of electric motors. Consequently, reliability assurance programs focused on motor health are supplanting the historic practices of maintaining motors but also allowing them to run until failure.

Several online fault monitoring techniques of varying complexity have been developed and deployed. Among the simple techniques are Negative Sequence Detectors [8], Kappa Transforms [16], Voltage Mismatch Detectors [18][14]. More advanced techniques of pattern classification have also been used, for example, Mechanical Vibration Monitoring [17], Signal Spectral Analysis [10], Fuzzy Logic and Neural Networks [13][3][4]. Both simple and advanced techniques have their respective drawbacks. Simple techniques may not provide a sufficient warning, while advanced techniques may require excessive computing resources. In this paper we take a middle path, a simple yet computationally robust method that provides early detection of imbalances. The framework is a blend of Signal Processing along with Symbolic Dynamics and Finite State Machines. The only assumptions made under this framework are :

- The process is stationary at the fast time scale.

- Any observable non-stationary behavior is associated with changes evolving at the slow time scale.

As we shall see, these assumptions do not limit the scope of the problem to be tackled. Instead they provide the means to detect imbalances at an incipient stage. It is assumed that the imbalance of the stator voltages increases with time (on the slow time scale).

$\dagger$ This work has been supported in part by Army Research Office (ARO) under Grant No. DAAD19-01-1-0646.
The framework consists of two parts

- Signal Processing : To extract information from the raw data we need to pre-process it. In this paper the Parks Vector transforms and Wavelet analysis were used to pre-process the raw data.

- Pattern Classification : After pre-processing the raw data, it is discretized to into finite number of intervals, where each interval is represented by a symbol. After these two steps we can see that the raw data has been changed to a symbol string. This symbol string contains information and this can be represented using a D-Markov machine.

These two parts of the framework to detect voltage imbalances are discussed in Sections III and IV after the basic characteristics of fault signatures are described in Section II.

\section{ANALYSis OF FAUlt SignAtures}

A basic analysis of the stator currents indicates that the fault information lies in the range of twice the line frequency. This differs from the case of rotor bar faults where the fault lies in the frequency region of $2 s f$ and $4 s f$, where $s$ is the slip and $f$ is the line frequency. To detect stator imbalances we start with the line currents represented by $i_{A}, i_{B}$ and $i_{C}$. Fault information is not readily discernable from the line currents, this is especially true for small values of imbalance. To isolate and identify the fault signature the Parks Vector transformation is used, which is nothing but a reference frame transform to a stationary frame. More specifically

$$
\begin{aligned}
i_{d} & =\frac{\sqrt{2}}{\sqrt{3}} i_{A}-\frac{1}{\sqrt{6}} i_{B}-\frac{1}{\sqrt{6}} i_{C} \\
i_{q} & =\frac{1}{\sqrt{2}} i_{B}-\frac{1}{\sqrt{2}} i_{C}
\end{aligned}
$$

where $i_{d}$ and $i_{q}$ are the direct and quadrature axis currents respectively. These currents should ideally be $\frac{\pi}{2}$ radians out of phase.

$$
\begin{aligned}
i_{d} & =\frac{\sqrt{6}}{2} i_{M} \cos (\omega t) \\
i_{q} & =\frac{\sqrt{6}}{2} i_{M} \sin (\omega t)
\end{aligned}
$$


In a parametric plot with $t$ as the parameter, the direct and quadrature axis currents represent a circle.

$$
i_{d}^{2}+i_{q}^{2}=\frac{3}{2} i_{M}^{2}
$$

when the load is a constant, $i_{M}$ should also be a constant. The quantity given in (5) is known as the Parks Vector Modulus. When there is an imbalance in the supply voltage, the above relations no longer hold true. The imbalanced supply voltage causes the currents to be unbalanced as well. The unbalanced currents are represented by positive sequence and negative sequence currents ${ }^{1}$ as, $i_{A}^{+}$and $i_{A}^{-}$. As shown in the Fig(1)

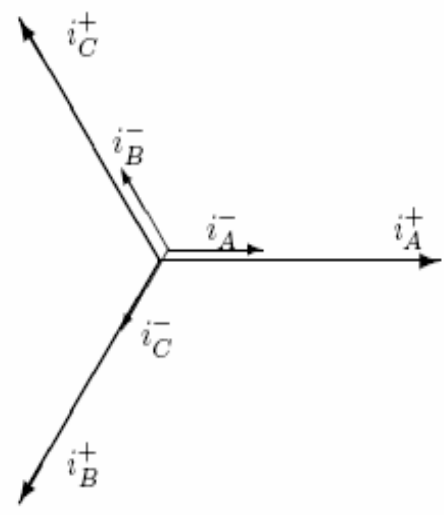

Fig. 1. Induction Motor Currents Under Imbalanced Operation

Let the positive and negative sequence currents have maximum values of $I_{+}$and $I_{-}$respectively. The three line currents can be represented by

$$
\begin{aligned}
i_{A} & =I_{+} \cos (\omega t)+I_{-} \cos (\omega t) \\
i_{B} & =I_{+} \cos \left(\omega t-\frac{2 \pi}{3}\right)+I_{-} \cos \left(\omega t-\frac{4 \pi}{3}\right) \\
i_{C} & =I_{+} \cos \left(\omega t-\frac{4 \pi}{3}\right)+I_{-} \cos \left(\omega t-\frac{2 \pi}{3}\right)
\end{aligned}
$$

Usually if the imbalance is not too large, then it is assumed that $I_{-} \ll I_{+}$. For this unbalanced condition, the direct and quadrature axis currents are [9]

$$
\begin{aligned}
& i_{d}=\left(I_{+}+I_{-}\right) \frac{\sqrt{6}}{2} \cos (\omega t) \\
& i_{q}=\left(I_{+}-I_{-}\right) \frac{\sqrt{6}}{2} \sin (\omega t)
\end{aligned}
$$

and the Parks Vector Modulus then becomes

$$
i_{d}^{2}+i_{q}^{2}=\frac{3}{2}\left(I_{+}^{2}+I_{-}^{2}\right)+3 I_{+} I_{-} \cos (2 \omega t)
$$

The fault signature clearly resides at twice the line frequency $2 \omega$. This can be confirmed by looking at the power spectra Fig(2).

The next few sections discuss in detail the framework employed to detect imbalances at an early stage. A block diagram of this is shown in $\operatorname{Fig}(3)$.

\footnotetext{
${ }^{1}$ This being a three-wire system with a floating neutral there are no 0 -sequence currents
}

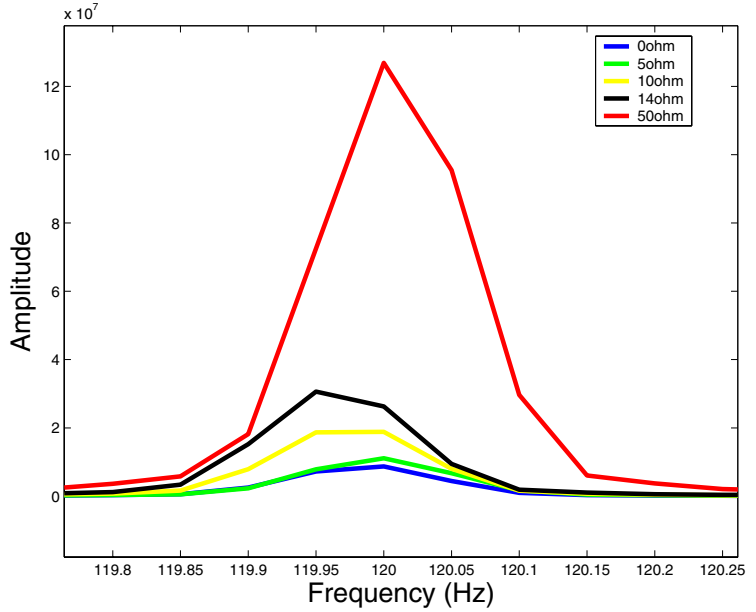

Fig. 2. Power Spectral Density

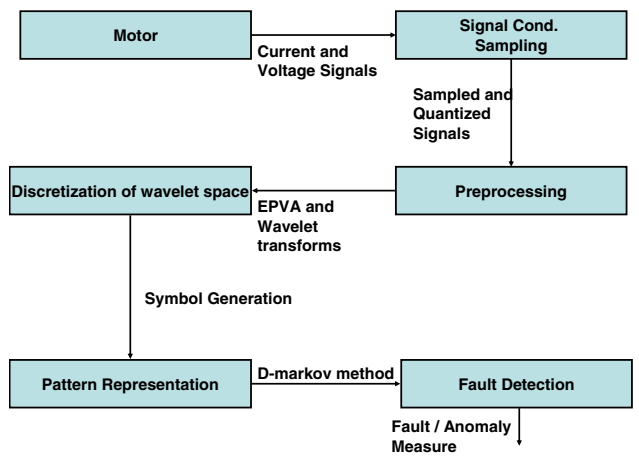

Fig. 3. Overall implementation diagram

\section{WaVelet Processing of Stator Signals}

Processing of the raw data or otherwise known as preprocessing, involves the collection of data, and the extraction of meaningful information from it. In this paper wavelet analysis is used, since provides both time and frequency information [2]. The continuous wavelet transform was used for the analysis, which for a function $f(t)$ is given by

$$
C W T_{\alpha, \beta}=\int_{-\infty}^{\infty} f(t) \psi^{*}\left(\frac{t-\beta}{\alpha}\right) d t
$$

where $\psi(t)$ is the mother wavelet with $\alpha>0$ being the scale and $\beta$ being the time shift. Hence, the wavelet transform coefficients are a function of both time and scale.

The choice of mother wavelet $\psi(t)$ and the scales $\alpha$, are crucial to the results of the fault detection methodology. If an inappropriate mother wavelet is chosen, the number of coefficients required to represent the original signal will be large. Several mother wavelets were tested including Haar, Gauss, and Mexican Hat. All of them performed satisfactorily. The Haar wavelet was chosen for its simplicity and for the fact that it can detect changes from a DC/constant value easily [11]. Similarly, there is no procedure for the choice 
of scales. However, the following guideline has proven to be useful.

For every wavelet, there exists a certain frequency called the center frequency $\left(F_{c}\right)$. The psuedo-frequency $\left(f_{p}\right)$ of the wavelet at a particular scale $\alpha$ is given by the following formula [5]

$$
f_{p}=\frac{F_{c}}{\alpha \cdot \Delta t}
$$

$\Delta t$ is the sampling interval and $\Delta t=1 / F_{s}$ where $F_{s}$ the sampling frequency. The Power Spectral Density (PSD) of the given signal or physical knowledge of the system from which the signal is obtained provides the information about the frequencies over which the fault signature is predominant. This information along with (13) can be used to choose the scales.

Once the mother wavelet and the scales are chosen, the wavelet coefficients are obtained for each of the scales. These coefficients are stacked from end to end starting with the smallest value of scale and ending with the largest value. For example, the wavelet coefficients versus scale at time shift $\beta_{k}$ are stacked after the ones at time shift $\beta_{k-1}$, to obtain the so-called scale series data in the wavelet space.

\section{A. Discretization of Wavelet Space}

After the scale series data is constructed, the objective is to convert this data into a sequence of symbols. A scheme for partitioning based on thresholds was attempted in [7]. In this method, the maximum and minimum of the scale series was found. The ordinates between the maximum and minimum were split into equal-sized regions. These regions are mutually disjoint and they form a partition. Each region is associated with one symbol from the alphabet. If the data point lies in a particular region, it is coded with the symbol associated with that region. Thus a sequence of symbols is generated from the scale series. Fig(4) depicts the partitions and the symbols associated with them.

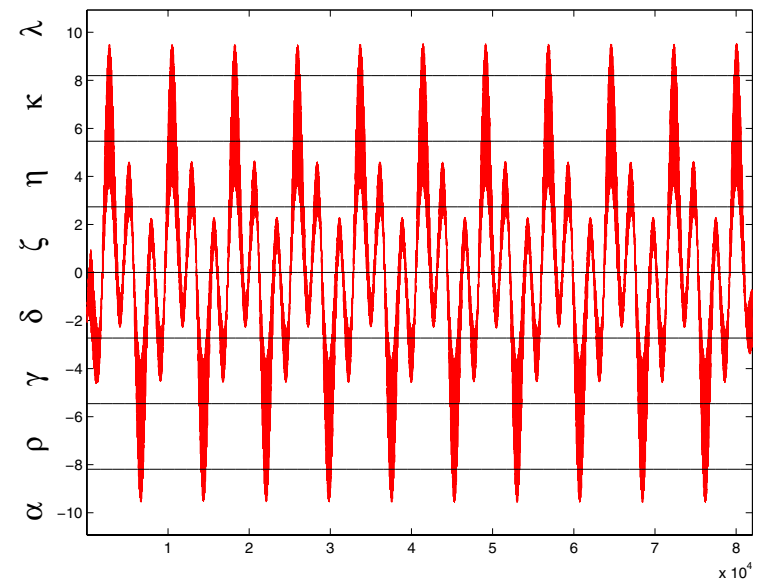

Fig. 4. Symbol Generation from Scale Series

\section{B. Motivation and methodology for partitioning}

As mentioned previously, modeling a physical process with the mathematical structure may not always be feasible. A convenient way of learning the dynamical behavior is to rely on the (sensor-based) observed behavior. The need to extract relevant physical information about the observed dynamics has lead to development of nonlinear time series analysis (NTSA) techniques. Identification of nonlinear systems can be achieved using Formal Languages. In this paper, a discrete symbolic description is derived from the scale series obtained from the wavelet analysis. The wavelet space is first discretized based on an appropriate Poincare section $\mathrm{P}$ [6], the wavelet transform is essentially a linear operator, hence, this is similar to partitioning the time series data. The resulting space of the map $f$ associated with the flow through $\mathrm{P}$ is divided into cells, so as to obtain a coordinate grid for the dynamics.

For simplicity, a compact set $\Omega \in \mathbb{R}^{n}$ within which the motion is circumscribed, is identified with the wavelet space itself. The encoding of $\Omega$ is accomplished by introducing a partition $\mathbf{B}=\left(B_{0}, B_{1}, B_{2}, B_{n-1}\right)$ consisting of $n$ mutually exclusive and exhaustive subsets, i.e., $B_{j} \bigcap B_{k}=\oslash, \forall j \neq k$ , $\bigcup_{j=1}^{n} B_{j}=\Omega$. The dynamical system describes an orbit $\mathrm{O}$ $=\left(x_{0}, x_{1}, x_{2}, \ldots \ldots x_{n}, \ldots\right)$, which passes through or touches various elements of the partition $\mathrm{B}$. Let us denote the index of domain $B_{i} \in \mathbf{B}$ visited at the time instant $i$ as the symbol $\sigma_{i} \in \Sigma$. The set of symbols $\Sigma=\left(\sigma_{1}, \sigma_{2} \ldots \ldots \sigma_{n-1}\right)$ labeling the partition elements is called the alphabet. This forms a mapping from the wavelet space to the space of symbols. Such a mapping is called Symbolic Dynamics, as it attributes a legal (i.e., physically admissible) symbol sequence to the system dynamics. As the size of each cell is finite and the cardinality of the alphabet $\Sigma$ is finite, any such symbol sequence represents, through iteration, a trajectory that has the compact support $\Omega$.

In general, a dynamical system would generate only a subset of all possible sequences of symbols as there are many illegal (i.e., physically inadmissible) sequences. The grammar (i.e., set of rules) that determines legality of symbol strings in the alphabet $\Sigma$ may change with the occurrence of the fault, which as discussed earlier occurs in the slow time scale. Symbolic Dynamics can be viewed as coarse graining of the wavelet space and hence subjected to (possible) loss of information. However,the essential robust features (e.g.,periodic behavior or chaotic behavior of an orbit) are expected to be preserved in the symbol sequences through an appropriate partitioning of the wavelet space [1]. In this paper the wavelet space (scale series) is partitioned into segments of equal size.

\section{PATtern ClassificAtion}

Having obtained the symbol sequence there arises a need to represent this pattern of symbols. This was done using a D-Markov machine. 


\section{A. D - Markov Machines}

After obtaining the symbol sequence, a finite state machine was constructed. The purpose of a finite state machine is to represent the dynamical behavior of the underlying process. In this paper, we use a kind of finite state automata, known as the D-Markov machine, to represent the symbolic process.

The core assumption here is that the symbolic process can be approximated, to a desired level of accuracy, as a $D^{t h}$ order Markov chain and $\mathrm{D} \in \mathbb{N}$, where $\mathbb{N}$ is the set of natural numbers. The states are chosen as words of length $\mathrm{D}$ from the symbol sequence. With the cardinality $|\Sigma|$ of the of the alphabet and the depth $\mathrm{D}$, the number of states in the $\mathrm{D}$-Markov machine is given by $|\Sigma|^{D}$. The state machine moves from one state to another upon occurrence of an event. All symbol sequences for which the last D symbols are the same, lead to the same state. For the case that $\mathrm{D}=1$, it becomes a regular Markov process, with each new symbol leading it to the next state. In this paper this happens to be the case and the D-Markov machine is shown in Fig(5).

Having constructed the D-Markov machine, the transitional probabilities $\pi_{i j}^{\left(\sigma_{\ell}\right)}$ between the states, upon occurrence of the symbol $\sigma_{\ell} \in \Sigma$, are determined, where $i$ is the state of origin and $j$ is the state transitioned to. As the system trajectory evolves, different states are visited with different frequencies. The number of times a state is visited as well as the number of times a particular symbol is received, is counted. The state probabilities as well as the state-state transition probabilities are calculated for each state in this way. The probabilities are obtained by frequency counts. Thus $\pi_{i j}^{\left(\sigma_{\ell}\right)} \approx \frac{n_{i}^{\left(\sigma_{\ell}\right)}}{N_{i}}$, where $n_{i}^{\left(\sigma_{\ell}\right)}$ is the number of times the symbol $\sigma_{\ell}$ occurred at the state $\mathrm{i}$ and $N_{i}$ is the total number of occurrences of symbols at the state i, i.e $N_{i}=\sum_{k} n_{i}{ }^{\left(\sigma_{k}\right)}$. The matrix comprised of all the transitional probabilities between the various states is called the state transition matrix $\Pi$, shown in Tables (I),(II) and (III). The columns of the matrices indicate the original state and the rows indicate the state transitioned to. The entries in the tables indicate the probabilities with which these transitions occur. The row sum of these $\Pi$ matrices should always be unity, since $\Pi$ is a stochastic matrix. The unity-valued entries in the matrix correspond to the states that are not visited at all, since if the machine does go into any of those states, it will not be able to exit the state.

\section{B. Anomaly Measure and Detection}

The machines described in Section(IV-A) are capable of representing patterns in the behavior of a dynamical system under faulty/anomalous conditions. In order to quantify changes in the patterns, we induce a measure on these machines, denoted as an anomaly measure $\mathbf{M}$.

The state transition probabilities are dependent on the dynamics of the complex system as reflected in the symbol sequence. This is a factor in detecting an fault/anomaly because perturbations in the system dynamics causes sig-

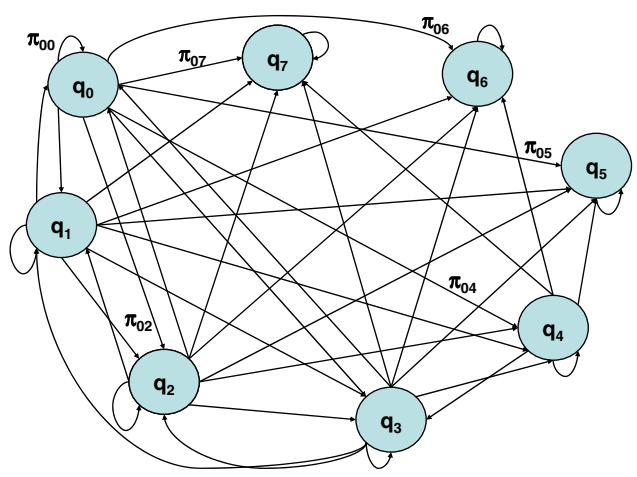

Fig. 5. Automata for the Induction Motor

nificant changes in the state transition probabilities, though such changes may also be dependent on the wavelet space is partitioned (if it is uniformly partitioned or not). The induced norm of the difference between the state transition matrices $\Pi_{0}$ and $\Pi$, respectively for the nominal and anomalous state machines $G_{0}$ and $G$, may be used as a measure of anomaly,i.e. $\mathbf{M}\left(G_{0}, G\right)=\left\|\Pi_{0}-\Pi\right\|$. Such a measure, adopted in [15], was found to be very effective. Alternatively, a measure of anomaly may be derived directly from state probability vector $\mathbf{p}$. The state probability vector $\mathbf{p}$ is the vector, whose elements denote the probability of occurrence of states of the D-Markov machine. This is obtained by computing the left eigenvector of the $\Pi$ matrix, corresponding to the eigen value 1.0. Since it has to satisfy the stationary Markov property

$$
\mathbf{p} \Pi=\mathbf{p}
$$

Table(IV) shows the state probability vector for three cases of 0,5 and $50 \mathrm{Ohm}$ imbalances respectively. Since DMarkov machines have a fixed state structure, the state probability vector associated with all state machines will have the same dimension. Hence they may be used for vector representation of the anomaly, leading to the anomaly measure $\mathbf{M}\left(G_{0}, G\right)=\left\|\mathbf{p}_{\mathbf{0}}-\mathbf{p}\right\|$.

TABLE I

II MATRIX FOR R = 0 OHMS

\begin{tabular}{|c||c|c|c|c|c|c|c|c|}
\hline & $q_{0}$ & $q_{1}$ & $q_{2}$ & $q_{3}$ & $q_{4}$ & $q_{5}$ & $q_{6}$ & $q_{7}$ \\
\hline \hline$q_{0}$ & 1.0000 & 0 & 0 & 0 & 0 & 0 & 0 & 0 \\
\hline$q_{1}$ & 0 & 1.0000 & 0 & 0 & 0 & 0 & 0 & 0 \\
\hline$q_{2}$ & 0 & 0 & 0 & 1.0000 & 0 & 0 & 0 & 0 \\
\hline$q_{3}$ & 0 & 0 & 0.0001 & 0.9475 & 0.0524 & 0 & 0 & 0 \\
\hline$q_{4}$ & 0 & 0 & 0 & 0.0569 & 0.9268 & 0.0163 & 0 & 0 \\
\hline$q_{5}$ & 0 & 0 & 0 & 0 & 0.2302 & 0.7698 & 0 & 0 \\
\hline$q_{6}$ & 0 & 0 & 0 & 0 & 0 & 0 & 1.0000 & 0 \\
\hline$q_{7}$ & 0 & 0 & 0 & 0 & 0 & 0 & 0 & 1.0000 \\
\hline
\end{tabular}

\section{EXPERIMENTAL RESUlts AND Discussion}

\section{A. Experimental Setup}

The experimental setup consists of the following 
TABLE II

П MATRIX FOR R = 5 OHMS

\begin{tabular}{|c||c|c|c|c|c|c|c|c|}
\hline & $q_{0}$ & $q_{1}$ & $q_{2}$ & $q_{3}$ & $q_{4}$ & $q_{5}$ & $q_{6}$ & $q_{7}$ \\
\hline \hline$q_{0}$ & 1.0000 & 0 & 0 & 0 & 0 & 0 & 0 & 0 \\
\hline$q_{1}$ & 0 & 1.0000 & 0 & 0 & 0 & 0 & 0 & 0 \\
\hline$q_{2}$ & 0 & 0 & 0.7972 & 0.2028 & 0 & 0 & 0 & 0 \\
\hline$q_{3}$ & 0 & 0 & 0.0464 & 0.8871 & 0.0665 & 0 & 0 & 0 \\
\hline$q_{4}$ & 0 & 0 & 0 & 0.0611 & 0.9093 & 0.0296 & 0 & 0 \\
\hline$q_{5}$ & 0 & 0 & 0 & 0 & 0.1832 & 0.8168 & 0 & 0 \\
\hline$q_{6}$ & 0 & 0 & 0 & 0 & 0 & 0 & 1.0000 & 0 \\
\hline$q_{7}$ & 0 & 0 & 0 & 0 & 0 & 0 & 0 & 1.0000 \\
\hline
\end{tabular}

TABLE III

II MATRIX FOR R $=50$ OHMS

\begin{tabular}{|c||c|c|c|c|c|c|c|c|}
\hline & $q_{0}$ & $q_{1}$ & $q_{2}$ & $q_{3}$ & $q_{4}$ & $q_{5}$ & $q_{6}$ & $q_{7}$ \\
\hline \hline$q_{0}$ & 0.8547 & 0.1094 & 0.0289 & 0.0069 & 0.0001 & 0 & 0 & 0 \\
\hline$q_{1}$ & 0.1453 & 0.7012 & 0.0956 & 0.0397 & 0.0179 & 0.0004 & 0 & 0 \\
\hline$q_{2}$ & 0.0473 & 0.1354 & 0.6406 & 0.1058 & 0.0434 & 0.0275 & 0 & 0 \\
\hline$q_{3}$ & 0.0077 & 0.0659 & 0.1181 & 0.6240 & 0.1139 & 0.0505 & 0.0200 & 0 \\
\hline$q_{4}$ & 0 & 0.0186 & 0.0579 & 0.1114 & 0.6263 & 0.1184 & 0.0579 & 0.0095 \\
\hline$q_{5}$ & 0 & 0 & 0.0174 & 0.0564 & 0.1047 & 0.6513 & 0.1335 & 0.0368 \\
\hline$q_{6}$ & 0 & 0 & 0 & 0.0089 & 0.0435 & 0.0998 & 0.7238 & 0.1240 \\
\hline$q_{7}$ & 0 & 0 & 0 & 0 & 0.0033 & 0.0223 & 0.1111 & 0.8632 \\
\hline
\end{tabular}

- Motors: 3-phase, 2-HP Y-connected squirrel cage induction motors were used. These motors are coupled using a flexible Lovejoy coupling to the dynamometer.

- Load: For loading the motors a hysteresis dynamometer was used, this provides two-quadrant operation. The dynamometer used was a model HD-805 6N Magtrol Inc, a hysteresis brake.

- Sensors : The sensor board consisted of both voltage and current sensors. The sensors have an extremely high bandwidth, they are intentionally kept that way so that high frequency as well as low frequency components harmonics could be measured and analyzed. The sensors used were LEM model numbers LV-25P and LA-55P . This is followed by operational amplifiers, realized by LM-324 IC's which provide sufficient gain. The amplified signal is sent to the data acquisition board.

- Processor and Data Acquisition: The data acquisition is the dSPACE Ds-1103 PPC card. This card has a Motorola Power PC $400 \mathrm{Mhz}$ processor. It also features 16, 14 bit ADC units and 4,12 bit ADC units, as well as 8 DAC outputs.

The imbalance was created by adding a resistor to one of the phases of the motor. The resistance was increased for every run, the values were $0,1,5,10,14$ and $50 \mathrm{Ohm}$. In the ideal case for no added resistance i.e. $0 \mathrm{Ohm}$ imbalance the plot should be circular as indicated by (5). But this is not the case as there is already exists a supply imbalance in the laboratory. However, we can see that the shape of the phase plots becomes more elliptical, with the increase in resistance. This is shown in $\operatorname{Fig}(6)$.

\section{B. Results and Discussion}

Fig(3) shows the experimental procedure followed in the laboratory. The motor was loaded to $1 \mathrm{~kW}$, which is about $3 / 4$ of the full load. The data was taken for 100 seconds at a sampling rate of $1 \mathrm{kHz}$. For analysis, 10 seconds of data
TABLE IV

p VECTOR FOR $\mathrm{R}=0$ OHMS, 5 OHMS AND 50 OHMS

\begin{tabular}{|c||c|}
\hline$q_{0}$ & 0 \\
\hline$q_{1}$ & 0 \\
\hline$q_{2}$ & 0.0001 \\
\hline$q_{3}$ & 0.5033 \\
\hline$q_{4}$ & 0.4637 \\
\hline$q_{5}$ & 0.0329 \\
\hline$q_{6}$ & 0 \\
\hline$q_{7}$ & 0 \\
\hline
\end{tabular}

\begin{tabular}{|c||c|}
\hline$q_{0}$ & 0 \\
\hline$q_{1}$ & 0 \\
\hline$q_{2}$ & 0.0918 \\
\hline$q_{3}$ & 0.4013 \\
\hline$q_{4}$ & 0.4365 \\
\hline$q_{5}$ & 0.0704 \\
\hline$q_{6}$ & 0 \\
\hline$q_{7}$ & 0 \\
\hline
\end{tabular}

\begin{tabular}{|c||c|}
\hline$q_{0}$ & 0.1711 \\
\hline$q_{1}$ & 0.1338 \\
\hline$q_{2}$ & 0.0996 \\
\hline$q_{3}$ & 0.0919 \\
\hline$q_{4}$ & 0.0933 \\
\hline$q_{5}$ & 0.1042 \\
\hline$q_{6}$ & 0.1424 \\
\hline$q_{7}$ & 0.1637 \\
\hline
\end{tabular}

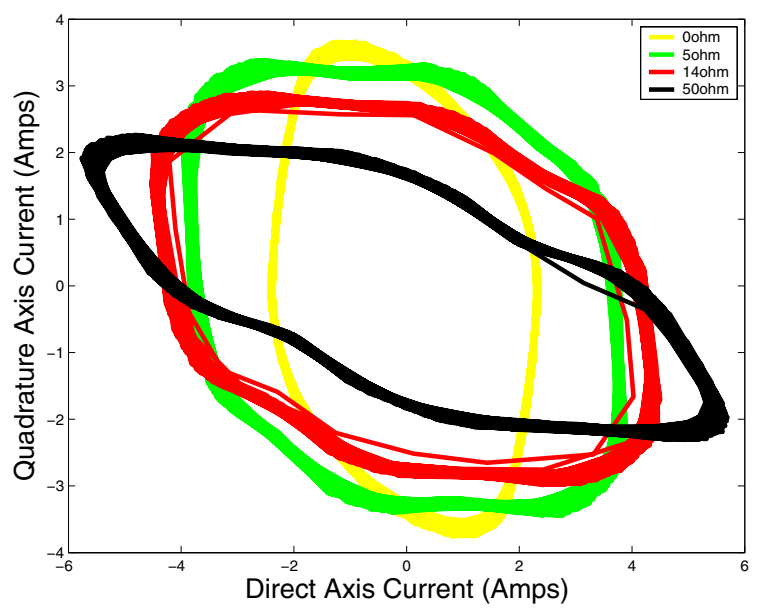

Fig. 6. Parks Vector plot

was used after the motor had reached steady-state operation. Other tests at various other load levels were also performed these include no-load and $0.5 \mathrm{~kW}$. All the other load levels also showed similar results except for the no-load test. In the no-load test, the currents were not high enough to show the fault signature. After this an online Parks Vector transform was computed. This was followed by the calculation of the Parks Vector Modulus, according to (11). The mean of this modulus is subtracted from each sample, this was done to remove the dc bias. Wavelet analysis was performed on this transformed data, the wavelet used was 'db1', the scales were chosen between 6 and 10 with a step size of 0.2 , using the guidelines given in the previous section(s). The alphabet size was 8 and the depth was chosen to be 1 . Table(I) gives the nominal $\Pi$ matrix, while the Table(II) and Table(III), show the $\Pi$ matrices for $5 \mathrm{Ohm}$ and 50 $\mathrm{Ohm}$ imbalances respectively. It can be clearly seen that the structure of these matrices changes dramatically, with the increase in imbalance. Several transitions, which had no chance of occurring; for example, from $q_{0}$ to $q_{1}$ for the nominal case, occur in the case of the $50 \mathrm{Ohm}$ imbalance. There is an important observation to make in the nominal case, which is the fact that many of states have a transition probability of 1 , while transitioning to themselves. These state rarely occur, and if they do occur the automata stays in them (deadlock states). For most real systems this does not take place, hence the process stays in the states that do 
not have self-transition probabilities of unity.

The norm of the difference between the nominal condition, which is the one without imbalance and the ones with imbalance gives us the the anomaly/fault measure. In this case the anomaly measure plot was normalized. This is shown in Fig(7), by using a suitable thresholding function (as shown in the figure) we can detect the fault early. For a similar kind of a fault, other methods like neural networks could detect a change only when the imbalance exceeded $0.2 \mathrm{pu}$ which in this case is $28 \mathrm{Ohms}$ as reported in [12]. The main advantages to using symbolics dynamics is the excellent noise rejection, noise is averaged out by repeatedly traversing the D-Markov machine. The method is also computationally simple, since it is essentially a counting process.

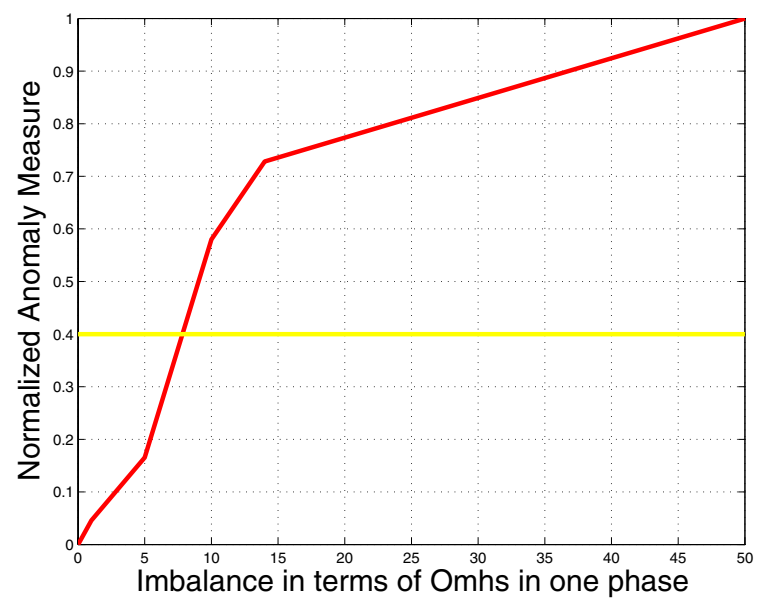

Fig. 7. Anomaly Measure

\section{CONCLUSIONS AND Future WORK}

This paper presented a new method for detecting voltage imbalances in Induction Motors. This technique is based on symbolic dynamics and wavelet analysis of stator current signals. A key advantage of this method is that it is computationally less intensive and yet provides scope for early detection of faults. The methodology was validated with experimental data obtained from a 3-phase, 2-HP squirrel cage induction motor. Future research is recommended in applying this methodology to other faults, like broken rotor bars. Also, we believe that this methodology can be applied to high performance drives, where both the noise levels and the bandwidth of the signals are higher.

\section{REFERENCES}

[1] R. Badii and A. Politi, Complexity hierarchical structures and scaling in physics, Cambridge University Press, United Kingdom, 1997.

[2] M.E.H. Benbouzid and G.B.; Kliman, What stator current processing-based technique to use for induction motor rotor faults diagnosis?, Energy Conversion, IEEE Transactions on, Volume: 18 , Issue: 2 pp.238 - 244, June 2003.

[3] M.-Y. Chow, R.N. Sharpe, and J.C. Hung, On the application and design of artificial neural networks for motor fault detection. $i$ and ii, Industrial Electronics, IEEE Transactions on , Volume: 40 , Issue: 2, April 1993.
[4] P.V. Goode and Mo yuen Chow, Using a neural/fuzzy system to extract heuristic knowledge of incipient faults in induction motors. part i-methodology, Industrial Electronics, IEEE Transactions on , Volume: 42 , Issue: 2, April 1995.

[5] Mathworks Inc, Wavelet toolbox - matlab.

[6] Hassan.K Khalil, Nonlinear systems, Prentice-Hall Inc, Englewood Cliffs, N.J., 1996.

[7] Amol Khatkhate, Asok Ray, Shin Chin, Venkatesh Rajagopalan, and Eric Keller, Detection of fatigue crack anomaly: A symbolic dynamics approach, American Control Conference, Boston, 2004.

[8] Jeffrey L. Kohler, Joseph Sottile, and Frederick C. Trutt, Condition monitoring of stator windings in induction motors: Part $i$ - experimental investigation of the negative-sequence impedance detector, IEEE Transactions on Industry Applications, vol. 38, No. 5, September/October 2002.

[9] Paul C. Krause, Oleg Wasynczuk, and Scott D. Sudhoff, Analysis of electric machinery, Piscataway, NJ : IEEE Press ; New York, NY : Institute of Electrical and Electronics Engineers, Inc, 1995.

[10] J.C. Kueck, J.D.and Criscoe and N.M. Burstein, Assessment of valve actuator motor rotor degradation by fourier analysis of current waveform, Energy Conversion, IEEE Transactions on , Volume: 7 , Issue: 3, Sept 1992.

[11] Stphane G Mallat; A wavelet tour of signal processing, 2/e, Academic Press, 1998.

[12] H. Nejjari and M.E.H.; Benbouzid, Monitoring and diagnosis of induction motors electrical faults using a current park's vector pattern learning approach, Electric Machines and Drives, 1999. International Conference IEMD '99 , 9-12 May, 1999.

[13] R.J. Patton, J. Chen, and C.J. Lopez-Toribio, Fuzzy observers for nonlinear dynamic systems fault diagnosis, Decision and Control, 1998. Proceedings of the 37th IEEE Conference on , Volume: 1 , 16-18, Dec. 1998.

[14] Joseph Sottile, Frederick C. Trutt, and Jeffrey L. Kohler, Condition monitoring of stator windings in induction motors: Part ii- experimental investigation of voltage mismatch detector, IEEE Transactions on Industry Applications, vol. 38, No. 5, September/October 2002.

[15] A. Surana, A. Ray, and S.C. Chin, Anomaly detection in complex systems, 5th IFAC Symposium on Fault Detection, Supervision and Safety of Technical Process, Washington, D.C., 2003.

[16] Frederick C. Trutt, Carlos Santa Cruz, Jeffrey L. Kohler, and Joseph Sottile, Prediction of electrical behavior in deteriorating induction motors, IEEE Transactions on Industry Applications, vol. 29, No. 6, November/December 1993.

[17] Frederick C. Trutt, Joseph Sottile, and Jeffrey L. Kohler, Detection of ac machine winding deterioration using electrically excited vibrations, IEEE Transactions on Industry Applications, Volume: 37 , Issue: 1, Jan.-Feb. 2001.

[18] _ Online condition monitoring of induction motors, IEEE Transactions on Industry Applications, Volume: 38 , No. 6, Nov-Dec 2002. 